\title{
Plasmacytoid dendritic cell subpopulations in head and neck squamous cell carcinoma
}

\author{
ANNETTE THIEL ${ }^{1}$, REBECCA KESSELRING ${ }^{1}$, RALPH PRIES ${ }^{1}$, \\ NADINE WITTKOPF ${ }^{1}$, ALEXANDER PUZIK $^{2}$ and BARBARA WOLLENBERG ${ }^{1}$ \\ ${ }^{1}$ Department of Otorhinolaryngology, University of Schleswig-Holstein Campus Lübeck, \\ 23538 Lübeck; ${ }^{2}$ Department of Pediatrics, University of Freiburg, 79106 Freiburg, Germany
}

Received December 16, 2010; Accepted January 19, 2011

DOI: $10.3892 /$ or.2011.1350

\begin{abstract}
Human plasmacytoid dendritic cells (PDCs) are present in solid tumor tissue and metastatic cervical lymph nodes (CLN) in head and neck squamous cell carcinoma (HNSCC). We recently showed that classical PDC functions are heavily disturbed in the tumor microenvironment. In this study we present a new approach to the subject by introducing 3 PDC subsets in HNSCC, characterized by the surface markers CD25, CD56 and CD203c. The first subset, positive for CD25, is significantly induced by HNSCC in vitro and present in metastatic lymph nodes in vivo. This subset can be phenotypically subdivided into matured cells and into a group expressing early $\mathrm{T}$ cell markers. Functionally this subgroup is associated with the secretion of IL- 8 . The second subset, positive for CD56, constitutes 4-5\% of all PDCs and is significantly down-regulated by HNSCC. Furthermore, this population sporadically expresses perforin/granzyme B and is absent in metastatic lymph nodes. The third subset, positive for the basophile marker CD203c, is inducible by crosslinking BDCA-2 in the presence of HNSCC and IL-4. Future studies will have to clarify the in vivo relevance of the different PDC subsets in HNSCC.
\end{abstract}

\section{Introduction}

Two main human dendritic cell subsets are to be distinguished: myeloid dendritic cells (MDCs) including langerhans cells, dermal dendritic cells and interstitial DCs versus plasmacytoid dendritic cells (PDCs) (1). Human PDCs are phenotypically defined by the expression of the surface markers CD123, BDCA-2 and HLA-DR. Furthermore, the classical PDC population is negative for the lineage markers

Correspondence to: Dr Barbara Wollenberg, Department of Otorhinolaryngology, University of Schleswig-Holstein-Campus Lübeck, Ratzeburger Allee 160, 23538 Lübeck, Germany

E-mail: barbara.wollenberg@uk-sh.de

Key words: plasmacytoid dendritic cell, subpopulations, head and neck squamous cell carcinoma
CD3, CD14, CD16, CD19, CD20, and CD56. Functionally, PDCs are to a small extent capable of presenting antigens to $\mathrm{T}$ cells (2), however, their main known function is characterized by the ability to produce large amounts of IFN- $\alpha$ in viral infections in vivo. In vitro, IFN- $\alpha$ secretion is also triggered by $\mathrm{CpG}$-oligonucleotides, which consist of unmethylated CpG-dinucleotides embedded in a certain sequence context within bacterial DNA $(3,4)$. It has been demonstrated that human solid HNSCC tumor tissues are infiltrated by plasmacytoid dendritic cells and that the classical PDC functions are heavily disturbed in the tumor microenvironment (5). Degraded to paralysed bystanders, the ability of secreting IFN- $\alpha$ is impaired whilst the migratory activity is enhanced (6). Hence, we present a new approach to the subject by introducing new PDC subpopulations in HNSCC. Recently, the existence of a CD2 positive PDC subset with functional autonomy and presence in different tumor tissues was reported (7). Future studies will have to clarify the in vivo relevance of the different PDC subsets in the tumor microenvironment and metastatic CLN.

\section{Materials and methods}

Isolation of PDCs. For in vitro studies plasmacytoid dendritic cells (PDC) were isolated from human peripheral blood provided by the blood bank of the University Hospital Lübeck, Germany. Blood donors were 18-65-year old healthy donors who were tested to be negative for allergies. PBMCs were obtained from buffy coats by Ficoll-Hypaque density gradient centrifugation as described elsewhere (8). PDCs were isolated by magnetic bead separation using magnetic labelled antiBDCA-4 antibodies (Miltenyi, Bergisch Gladbach, Germany). Tumor tissue and patient's blood were obtained during operations of tumor patients based on approval from the ethics committee, Campus Lübeck. Informed consent was obtained from each patient.

Single-cell suspensions. Metastatic lymph nodes were cut into pieces and then covered with $31.5 \mathrm{mg}$ collagenase $/ \mathrm{ml}$ PBS and $3.99 \mathrm{mg} / \mathrm{ml}$ hyaloronidase/ml PBS in DMEM and incubated in a $37^{\circ} \mathrm{C}$ water bath for 30-120 min. Digestion was checked every $30 \mathrm{~min}$. In a further step $33.4 \mathrm{mg}$ dispase $/ \mathrm{ml}$ PBS was added for 30-60 min. Brefeldin A (eBioscience, San 
A
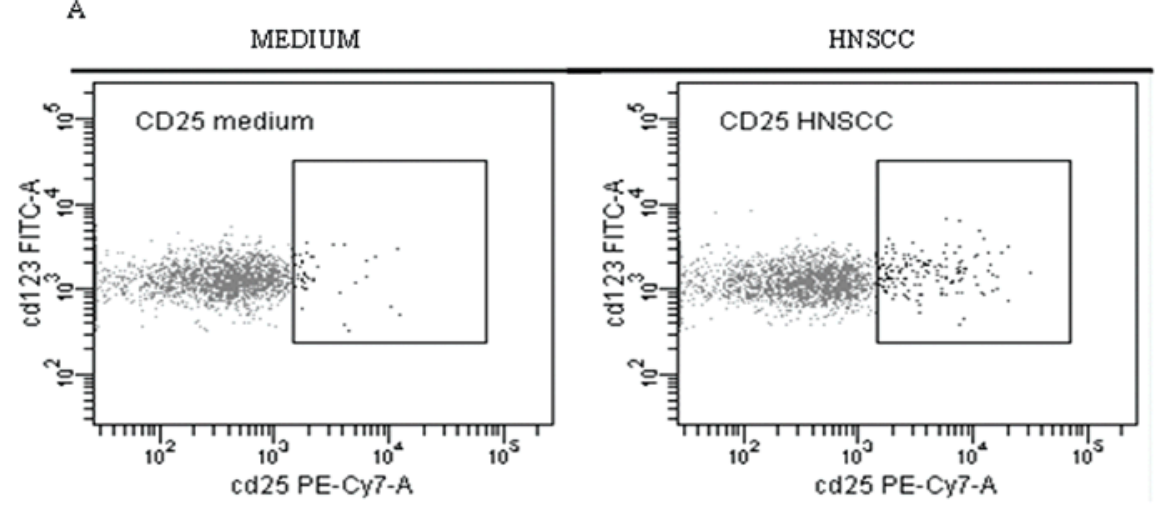

B

BDCA2-CY2

CD25-CY3

overlay + DAPI
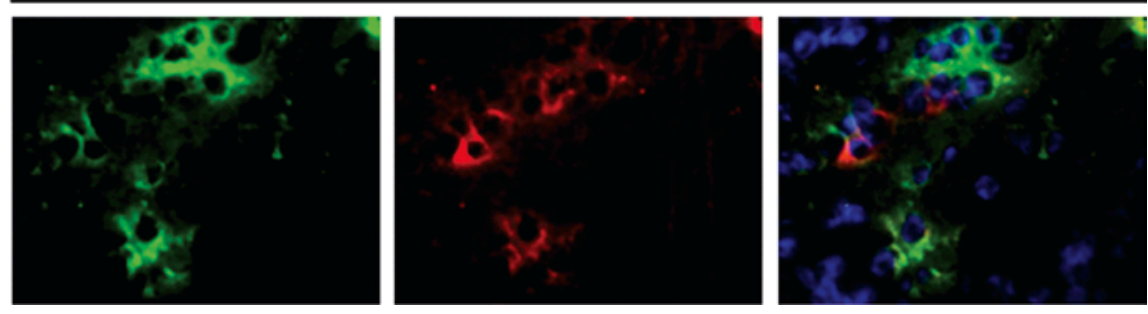

Figure 1. (A) CD25 positive subpopulation (5-20\% of the main PDC population, $\mathrm{p}=0.009$ ) was significantly induced after stimulation with the HNSCC cell lines PCI-1, PCI-13 and BHY in vitro. (B) Fluorescence staining of BDCA2 (green, CY2) and CD25 (red, CY3), in a metastatic lymph node.

Diego, CA, USA) was supplied during all steps. The digest was then passed through a $100-\mu \mathrm{m}$ nylon mesh in order to remove clumps and washed several times.

Cell cultures. The permanent HNSCC cell lines BHY (DSMZ Germany), PCI-1 and PCI-13 (generously provided by Professor T. Whiteside, Pittsburgh Cancer Institute) were cultured in Dulbecco's modified Eagle's medium (DMEM, Gibco, New York, NY, USA) supplemented with $10 \%$ FCS, $1 \mathrm{mM}$ glutamine, and $0.1 \mathrm{mM}$ sodium pyruvate. All compounds were purchased Endotoxin tested.

Fluorescence staining. Slices $(6 \mu \mathrm{m})$ were cryo-sectioned and placed on an object holder. The object holders were then dried for $10 \mathrm{~min}$, incubated in acetone for further $10 \mathrm{~min}$ and dried again for $10 \mathrm{~min}$ before washing them in PBS. In a further step the tissue samples were incubated with $30 \%$ goat serum (Vector) in PBS for at least $1 \mathrm{~h}$. Then the primary antibodies (CD25, IgG1, mouse, Imgenex and BDCA-2, IgG1, mouse, Miltenyi) and the isotype control (IgG1, mouse, Dianova) were each transferred onto the slices in $2 \%$ goat serum at a concentration of 1:50 for $2 \mathrm{~h}$ (room temperature). Before adding the second of two primary antibodies, the first one was blocked with goat anti-mouse FAB-Fragments (Jackson immunoResearch) according to the Dianova protocol. Subsequent to washing with PBS for 15 min the object holders were incubated with secondary antibodies (Cy3, donkey anti-goat and Cy2, goat anti-mouse, Jackson immunoResearch) for $30 \mathrm{~min}$ in $10 \%$ goat serum. After another 15-min washing step the tissue was stained with DAPI for $1 \mathrm{~min}$ and then covered with Fluromount. The expression of BDCA2 and CD25 in lymph node-tissue was analyzed on a Zeiss Axiovert 200M.
Flow cytometry. PDCs were identified as CD123-PE positive, HLA-DR-PerCP positive, lineage cocktail-FITC (CD3, CD14, CD16, CD19, CD20, CD56; Becton-Dickinson, Heidelberg, Germany) negative and BDCA2-APC/PE (Miltenyi Biotec, Bergisch-Gladbach, Germany) positive cells. Surface antigen staining was performed using the antibodies CD1aFITC, CD2-PE, CD3-PE, CD4-PERCP, CD4-APC-CY7, CD8-ARC-CY7, CD-25-APC/APC-CY7, CD34-PERCP, CD44-PE, CD56-PE, CD80-FITC, CD83-FITC, CD86-PE, CD117-APC, perforin-FITC, granzyme B-FITC, CD123-APC/ FITC, BDCA4-APC, CD45RA-FITC, CD25-FITC/PE-CY7, CD127-FITC (eBioscience) and GITR-FITC, IL-2 FITC, IL-4-PE, IL-6-FITC, IL-8-FITC, IL-10-APC, TGF- $\beta$-PE, TNF- $\alpha$-FITC (R\&D Systems, Minneapolis, MN, USA). The isotype PE $\operatorname{IgG} 1 / \operatorname{IgG} 2 \mathrm{a}$, the isotype APC IgG1/IgG2a and the isotype Fitc IgG1/IgG2b (Becton-Dickinson) were used as negative controls. Early apoptotic or dead cells were detected by staining the cells with Annexin-V-FITC and propidiumiodide-PE (Becton-Dickinson). Doublets were excluded by gating FSC-A against FSC-H. Only singlets were analysed. CD107a was up-regulated in the PDC population subsequent to stimulation with HNSCC, indicating cytokine secretion.

The amount of $2 \times 10^{5}$ PDCs was incubated on ice in $50 \mu 1$ $1 \%$ BSA (in PBS) with 1-2 $\mu \mathrm{l}$ of each antibody for $15 \mathrm{~min}$. Then the cells were washed three times in $500 \mu 1$ 1\% BSA and subsequently analysed on a FACSCanto (Becton-Dickinson) equipped with FACSDIVA software. Intracellular staining was performed with the eBioscience staining buffer set according to the eBioscience protocol. Cell sorting was performed with a Dako moFlow.

Cytokine analysis. The Bio-Plex Cytokine Assay (Bio-Rad, München, Germany) was used for the determinations of 
A
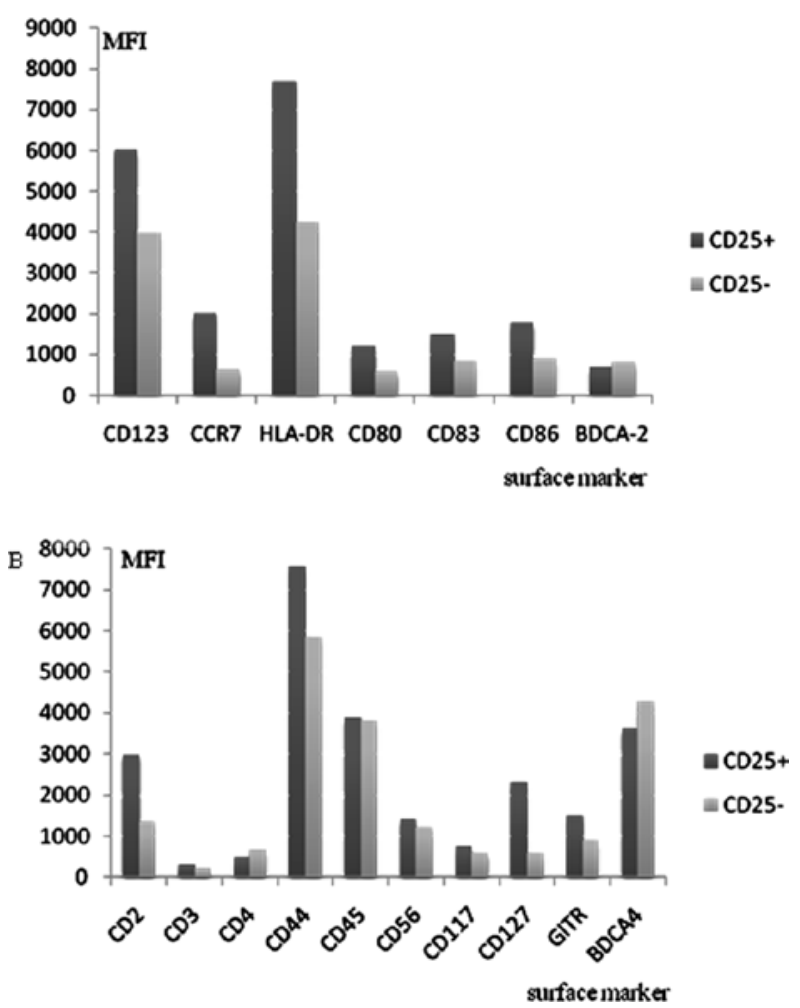

Figure 2. (A) CD25 positive subpopulation (40\%) is matured on the contrary to the CD25 negative PDC population after stimulation with HNSCC cell lines in vitro. (B) The MFI and percentage of cells positive (data not shown) for $\mathrm{T}$ cell and thymocyte markers is elevated in the CD25 positive subpopulation. We detected higher levels of CD2, CD3, CD38, CD44, CD45RA, CD117 (c-kit), CD127 and GITR. CD4- and BDCA4-levels were decreased when compared to the main PDC population. CD56 positive cells were upregulated in the CD25-subpopulation. *Two or three identical experiments were performed for each marker.

various cytokines such as interleukin-2 (IL-2), IL-4, IL-6, IL- 8 , IL-10, IL-12, IL-13, GM-CSF, IFN- $\gamma$ and TNF- $\alpha$. These cytokine assays allow the multiplexed quantitative measurement of multiple cytokines in a single small volume of cell culture supernatant. The protein array was analysed by a specialized microplate reader system (Bio-Plex Array Reader; Bio-Rad), and the data were calculated by the Bio-Plex Manager software.

Migration assay and MTT test. The cell line BHY (4.8x10 cells) was subjected to migration analysis using the ChemoTx system (NeuroProbe Inc., Gaithersburg, MD, USA). The microplate provided bottom wells for the chemoattractant IL-8. The cell suspensions were pipetted directly onto the sites on the top side of the filter sit and in hemispherical drops during incubation. Relative numbers of migrated cells were determined after $12 \mathrm{~h}$ using the MTT assay (In Vitro Toxicology Assay Kit, MTT-based; Sigma ${ }^{\circledR}$-Aldrich Co., Steinheim, Germany).

Statistical analysis. The Kolmogorow-Smirnow test did not show a normal distribution. Therefore, we performed the Wilcoxon-Mann-Withney test for statistical analysis. $\mathrm{p}<0.05$ was considered to be marginally significant.
A

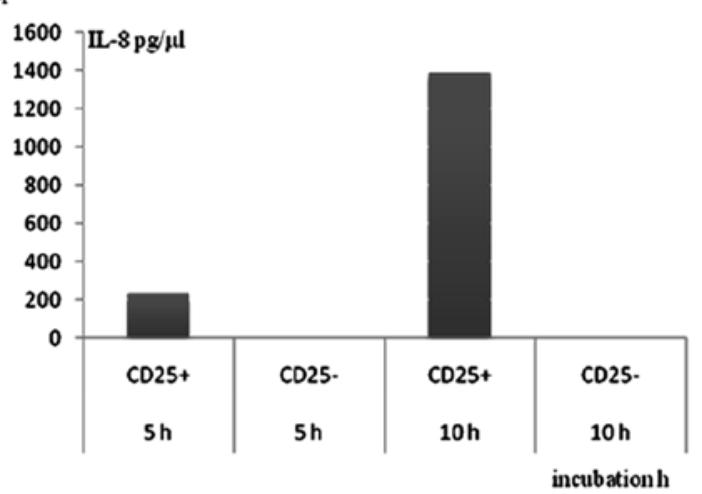

B

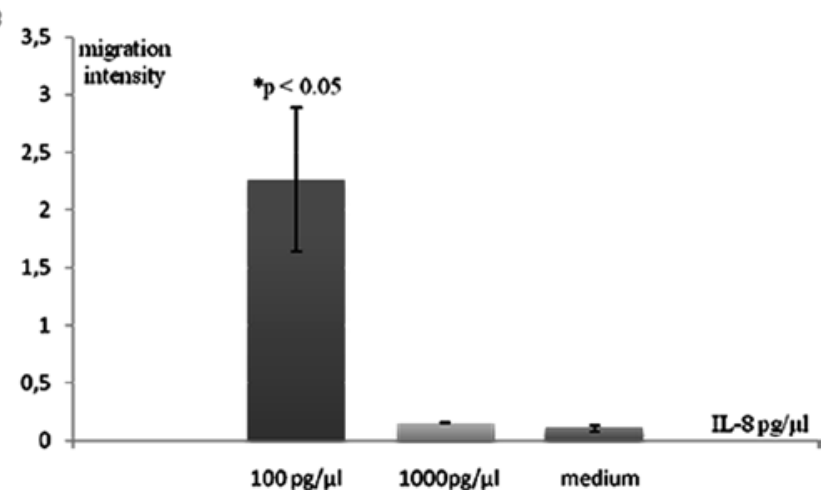

Figure 3. (A) Secretion of IL-8 above the levels of the HNSCC cell lines in the $\mathrm{CD} 25$ positive subpopulation after cell sorting. ${ }^{*}$ Two identical experiments were performed. (B) The HNSCC cell line BHY migrated towards IL-8 (100 pg/ $\mu 1)$ in vitro. *Three identical experiments were performed.

\section{Results}

HNSCC induces a CD25 positive subpopulation exhibiting a mature phenotype, besides an early T-cell phenotype. The significant induction $(\mathrm{p}=0.009)$ of a $\mathrm{CD} 25$ positive PDC subpopulation was observed after stimulation with HNSCC (Fig. 1A). Furthermore, this subset was present in metastatic CLN (Fig. 1B).

Having been described as the early activation marker on PDCs (9), we analysed if tumor-induced CD25 positive PDCs would likewise evoke a mature phenotype. Subsequent to the stimulation with HNSCC cell lines for $12 \mathrm{~h}$ the expression of the maturation markers CD123, CCR7, BDCA2, CD80, CD83, CD86 and HLA-DR in the CD25-subpopulation was compared to the classical PDC population. A higher expression of the surface markers CD123, CCR7, CD80, CD83 and CD86 besides a distinct down-regulation of BDCA-2 was observed on $40 \%$ of the CD25-subpopulation when compared to the CD25 negative PDCs (Fig. 2A). The remaining CD25 positive cells showed higher expression levels for some of the following $\mathrm{T}$ cell markers when compared to the CD25 negative PDC population subsequent to incubation with HNSCC cell lines for $12 \mathrm{~h}$. Following receptors were analysed: early $\mathrm{T}$ cell markers (CD1a, CD34, CD38, CD44, CD45, CD117 and CD127), common T cell markers (CD2, CD3, CD4) and Treg markers (GITR, CTLA-4) (Fig. 2B). 
A
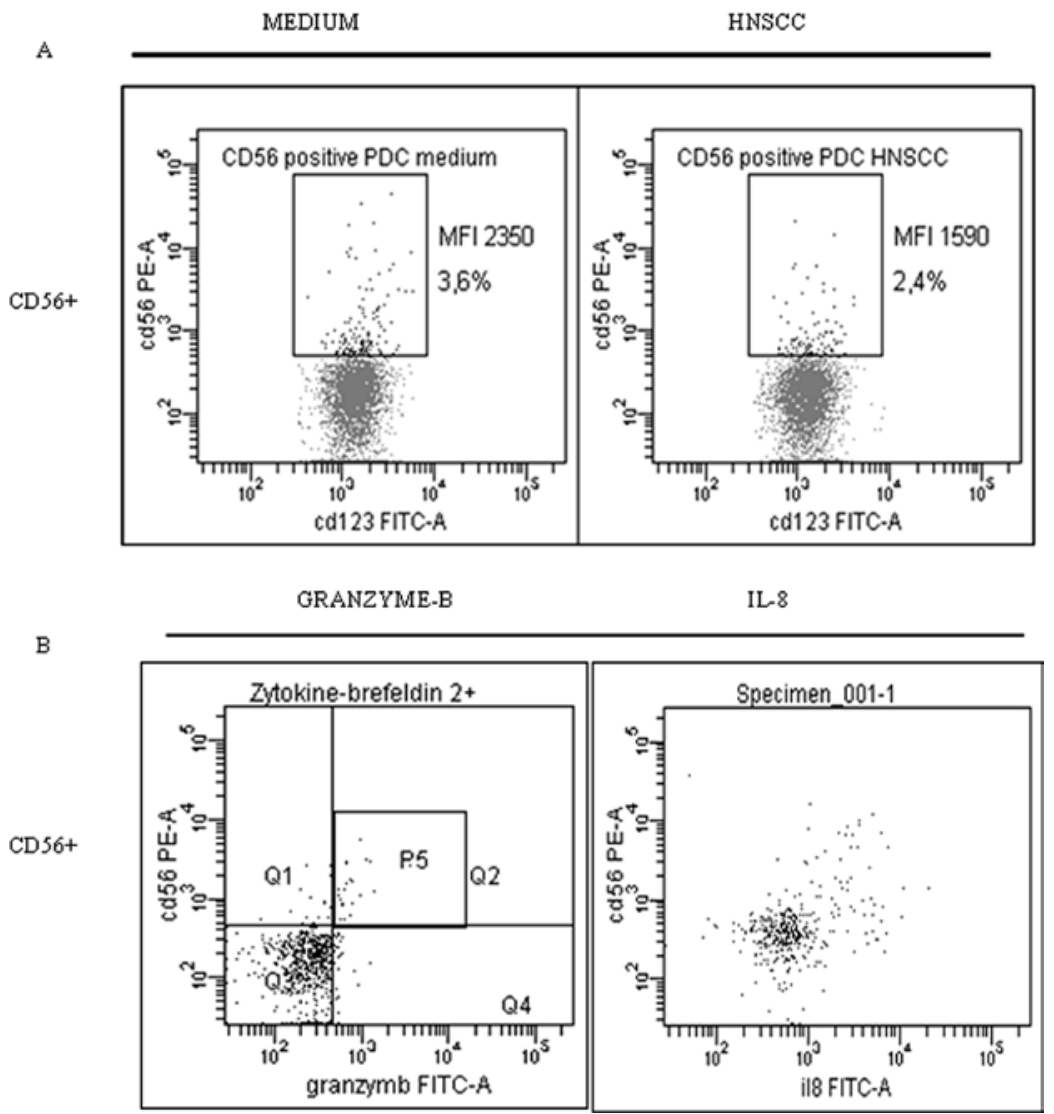

Figure 4. (A) We observed a significant ( $\mathrm{p}=0.0007$ ) down-regulation of the MFI and percentage of population in CD56 positive PDCs in healthy donors after stimulation with HNSCC. (B) Secretion of granzyme B and IL-8 in the CD56 positive subset.

The CD25 positive PDC subset reveals distinct functional properties. A Bio-Plex assay was performed after having sorted the CD25-subpopulation subsequent to incubation with HNSCC cell lines for $12 \mathrm{~h}$. After $8 \mathrm{~h}$ of further incubation with HNSCC supernatants and medium alternatively, IL-6 and IL-8 were likewise detected in the supernatants of the CD25-subpopulation. The CD25-subpopulation secreted IL-8 to a greater extent than the control, whilst secretion of IL-6 could not be distinguished from the amounts produced by the tumor cell lines. We could not detect any cytokine production above the HNSCC controls in the CD25-negative PDC population after $8 \mathrm{~h}$ (Fig. 3A).

In vivo a significant IL-8 production could not be referred to a single subpopulation, because of being secreted alternatively by the CD25 positive, a CD56 positive and a phenotypically as yet unknown subpopulation which could not be classified. No significant differences could be found concerning intracellular cytokine production (IL-2, IL-4, IL-6, IL-8, IL-10, TNF- $\alpha$, TGF- $\beta$ ) in the CD25 positive subpopulation and the CD25 negative PDC population in vivo.

In addition, in vitro investigations showed that the HNSCC cell line BHY migrated actively towards IL-8 $(100 \mathrm{pg} / \mu \mathrm{l})$ in a period of $12 \mathrm{~h}$ (Fig. 3B).

HNSCC significantly down-regulates CD56 positive PDCs. The PDC population (4-5\%) shows a CD56 positive phenotype (also partly positive for CD8) in healthy donors. In our study, we showed that this subpopulation was significantly $(\mathrm{p}=0.0007)$ down-regulated after incubation with the HNSCC cell line BHY (Fig. 4A). Although CD56 positive PDCs could be detected in the blood of tumor patients, we did not find a significant difference concerning the intracellular production of the cytokines IL-2, IL-4, IL-6, IL-8, IL-10 and TGF- $\beta$ in comparison to the classical PDCs. In vitro, sporadic expression of perforin, granzyme-B, TNF- $\alpha$ and IL- 8 was observed (Fig. 4B). CD56 positive PDCs were not present in metastatic lymph nodes.

BDCA-2 crosslinking in the presence of $I L-4$ and HNSCC induces a CD203c-positive PDC subpopulation. Besides the discovery of alterations concerning the CD25 and CD56 positive PDC subsets, we also observed the induction of a CD203c positive PDC subset in HNSCC. However, this population was not inducible by sole incubation with HNSCC. Crosslinking of the receptor BDCA-2 and addition of the cytokine IL-4 was mandatory for up-regulation of this surface marker. Furthermore, incubation with HNSCC and one of the mandatory components did not show any effect. BDCA-2 crosslinking in the presence of IL-4 in medium control induced a few CD203c positive PDCs, however, to a far lesser extent (Fig. 5).

\section{Discussion}

PDCs are to a small extent capable of presenting antigens to T cells (2), however, their main known function is characterized 


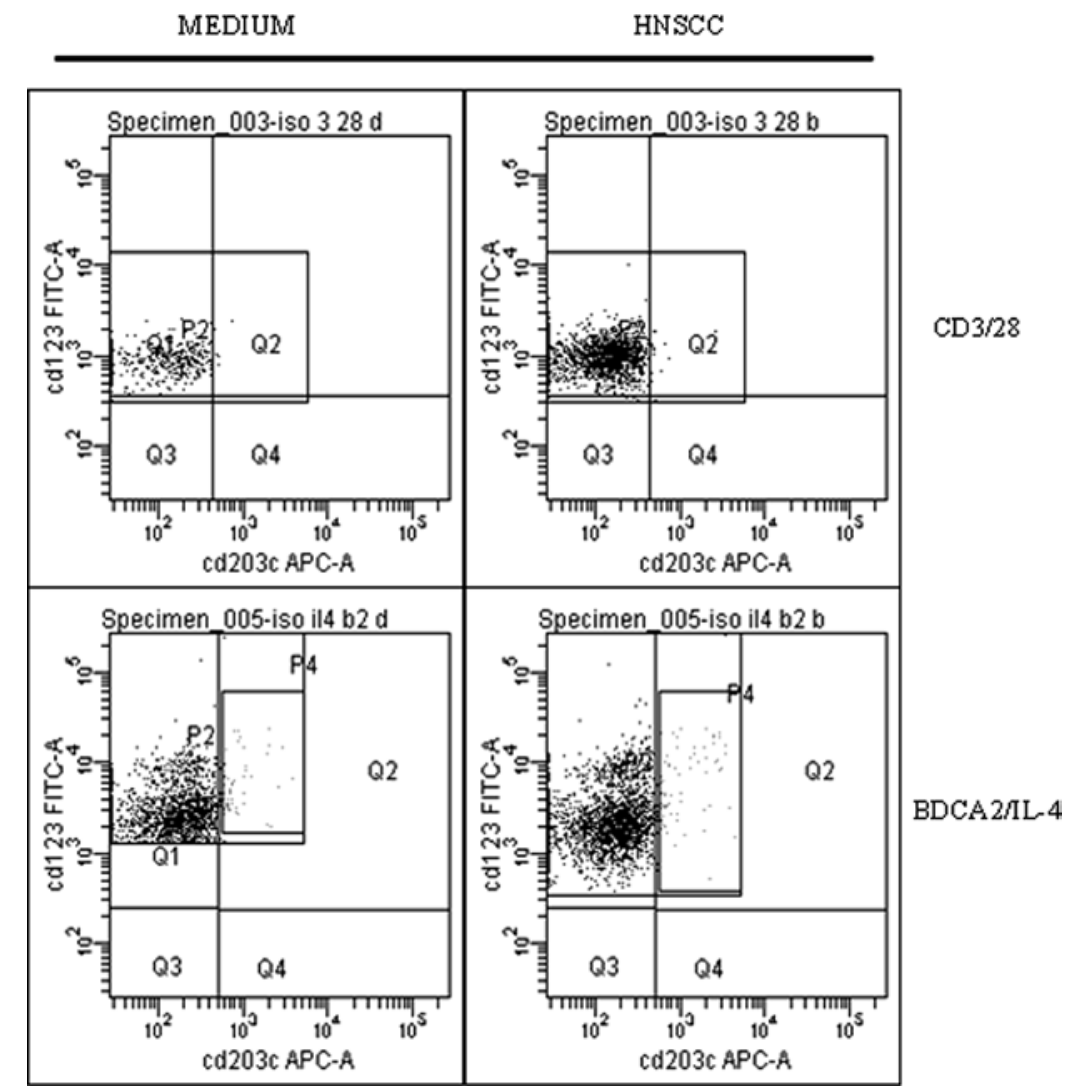

Figure 5. The new PDC subset positive for the Basophile marker CD203c was most effectively induced by crosslinking BDCA-2 in the presence of HNSCC and and IL-4 for $12 \mathrm{~h}$. Sole incubation with HNSCC, or crosslinking CD3/CD28 did not induce the CD203c-positive subset. "Three identical experiments were performed.

by the ability to produce large amounts of IFN- $\alpha$ in viral infections in vivo. In vitro, IFN- $\alpha$ secretion is also triggered by $\mathrm{CpG}$-oligonucleotides, which consist of unmethylated CpG-dinucleotides embedded in a certain sequence context within bacterial DNA $(3,4)$. We have recently shown that crucial PDC functions are impaired in HNSCC (6). Here we provide a new approach to the subject by focusing on PDC subsets in HNSCC.

HNSCC has an impact on at least three PDC subsets, which are characterized by the surface markers CD25, CD56 and CD203c.

The CD25 positive subpopulation was significantly induced by HNSCC in vitro and present in metastatic lymph nodes. In contrast to the CD25 negative PDCs, which showed an impaired maturation subsequent to incubation with HNSCC (unpublished data) (10), the CD25 positive subset partly exhibited a classically maturated phenotype as described before (9). However, the colocation of the up-regulation of CD4, CD45RA in the PDC population subsequent to stimulation with HNSCC (unpublished data) besides the up-regulation of CD25 in this specific subset, led us to consider a totally different direction. Due to the fact that $\mathrm{CD} 4$ and $\mathrm{CD} 45$ are associated with the modulation of the $T$ cell receptor (11), enhancement of positive selection (12) and pre-TCR signaling in thymocytes (13) besides CD123 being associated with early levels of differentiation (14) when highly expressed, we developed the assumption that these PDCs may be associated with peripheral early $\mathrm{T}$ cells. Indeed, those receptors which are elevated in the double negative stages 1-3 were partly up-regulated in the CD25 positive subpopulation, with exception of CD34 and CD1a. Only BDCA4 and CD4 were down-regulated when compared to the main PDC population. As down-regulation of CD25 is co-regulated with the up-regulation of BDCA-4 in murine thymocytes (15), the decrease of BDCA-4 may be linked to the up-regulation of CD25 in the HNSCC environment.

With exception of IL-8, which was secreted by the CD25 positive subset after exposure to HNSCC, we were confronted with major difficulties in distinguishing between cytokine-secretion by PDCs and HNSCC supernatants in the Bio-Plex assay, as HNSCC cytokine levels overlapped the production of cytokines by the PDC population. Furthermore, as supernatants containing few HNSCC cells were more effective in stimulating the PDCs than pure supernatants it was impossible to relate cytokine secretion to the different populations by Bio-Plex. Examining these results critically, the secretion of IL- 8 may therefore be alternatively induced in the tumor cell population by the CD25 positive subset. Interestingly, secretion of IL-8 is not maintained by a single population in vivo. The relevance of IL-8 being alternatively secreted by mature CD25 positive PDCs, a CD56 positive subset, an unclassified subpopulation and even the tumor cells themselves subsequent to stimulation with the CD25subpopulation remains to be resolved. In any case, IL- 8 expression correlates with the angiogenesis, tumorigenicity, and metastasis of many tumors and is supposed to confer 
chemotherapeutic resistance in cancer cells (16-18). These statements are underlined by the strong migratory activity towards IL-8 in the HNSCC cell line BHY in vitro.

The second PDC subset, which is characterized by expression of CD56 is significantly down-regulated subsequent to stimulation with HNSCC and is absent in metastatic lymph nodes. As this population sporadically displayed higher levels of perforin and granzyme-B when compared to the main PDC population, we suggest cytotoxic properties for this population. This subset may very well be associated with the murine IFN-producing killer dendritic cells (19) just as the monocyte derived CD56 ${ }^{+}$dendritic cells (20) being down-regulated after stimulation with HNSCC cell lines and accordingly absent in metastatic lymph nodes in vivo.

The third subset is represented by CD203c positive PDCs and was induced by crosslinking BDCA-2 in the presence of IL-4 and HNSCC. Originally this marker was additionally used in order to exclude the basophile subset. CD203c is a glycosylated type II transmembrane molecule that belongs to the family of ecto-nucleotide pyrophosphatase/ phosphodiesterase (E-NPP3) enzymes. Among hematopoietic cells, expression of CD203c is restricted to basophiles, mast cells and their precursors, and has been described as specific for this lineage (21). Protein and/or mRNA expression of CD203c has also been found in solid tissues such as uterus or prostate (22). As CD203c has recently been demonstrated in human colon carcinoma such as neoplastic bile disease and is associated with malignant subversion and invasive properties $(23,24)$, we propose a tumor benefitting role for the CD203c positive PDC subset in HNSCC. However, due to the size of the population, a functional analysis of this subset was not possible at present.

Future investigations and in vivo models will have to prove these theories and the clinical relevance for these PDC subsets in vivo.

\section{Acknowledgments}

We thank Tillmann Vollbrandt for skillful support in some parts of this study. We are grateful to all members of the Department of Otorhinolaryngology for their help. This study was supported by grants of the Mildred Scheel-Stiftung (Deutsche Krebshilfe), the Werner-and-Klara-Kreitz-Stiftung, the Monika-Kutzner-Stiftung and the Rudolf-BartlingStiftung.

\section{References}

1. McKenna K, Beignon AS and Bhardwaj N: Plasmacytoid dendritic cells: linking innate and adaptive immunity. J Virol 79: 17-27, 2005.

2. Tel J, Lambeck AJ, Cruz LJ, Tacken PJ, de Vries IJ and Figdor CG: Human plasmacytoid dendritic cells phagocytose, process, and present exogenous particulate antigen. J Immunol 184: 4276-4283, 2010.

3. Rothenfusser S, Tuma E, Endres S and Hartmann G: Plasmacytoid dendritic cells: the key to CpG. Hum Immunol 63: 1111-1119, 2002.

4. Meyers JH, Justement JS, Hallahan CW, et al: Impact of HIV on cell survival and antiviral activity of plasmacytoid dendritic cells. PLoS One 2: e458, 2007.
5. Hartmann E, Wollenberg B, Rothenfusser S, Wagner M, Wellisch D, Mack B, Giese T, Gires O, Endres S and Hartmann G: Identification and functional analysis of tumor-infiltrating plasmacytoid dendritic cells in head and neck cancer. Cancer Res 63: 6478-6487, 2003.

6. Thiel A, Pries R, Jeske S, Trenkle T and Wollenberg B: Effect of head and neck cancer supernatant and $\mathrm{CpG}$-oligonucleotides on migration and IFN-alpha production of plasmacytoid dendritic cells. Anticancer Res 29: 3019-3025, 2009.

7. Matsui T, Connolly JE, Michnevitz M, et al: CD2 distinguishes two subsets of human plasmacytoid dendritic cells with distinct phenotype and functions. J Immunol 182: 6815-6823, 2009.

8. Hartmann G, Krug A, Eigler A, Moeller J, Murphy J, Albrecht R and Endres S: Specific suppression of human tumor necrosis factor-alpha synthesis by antisense oligodeoxynucleotides. Antisense Nucleic Acid Drug Dev 6: 291-299, 1996.

9. Naranjo-Gomez M, Oliva H, Climent N, Fernandez MA, Ruiz-Riol M, Bofill M, Gatell JM, Gallart T, Pujol-Borrell R and Borras FE: Expression and function of the IL-2 receptor in activated human plasmacytoid dendritic cells. Eur J Immunol 37: 1764-1772, 2007.

10. Perrot I, Blanchard D, Freymond N, Isaac S, Guibert B, Pacheco Y and Lebecque S: Dendritic cells infiltrating human non-small cell lung cancer are blocked at immature stage. J Immunol 178: 2763-2769, 2007.

11. Sato T, Hozumi K, Kishihara K, Kametani Y, Sato C, Kumagai Y, Mak TW and Habu S: Evidence for down-regulation of highly expressed TCR by CD4 and CD 45 on non-selected CD4 ${ }^{+} \mathrm{CD} 8^{+}$ thymocytes. Int Immunol 8: 1529-1535, 1996.

12. Ong CJ, Dutz JP, Chui D, Teh HS and Marth JD: CD45 enhances positive selection and is expressed at a high level in large, cycling, positively selected $\mathrm{CD} 4{ }^{+} \mathrm{CD} 8^{+}$thymocytes. Immunology 91: 95-103, 1997.

13. Pingel S, Baker M, Turner M, Holmes $\mathrm{N}$ and Alexander DR: The CD45 tyrosine phosphatase regulates CD3-induced signal transduction and $\mathrm{T}$ cell development in recombinase-deficient mice: restoration of pre-TCR function by active p56(lck). Eur J Immunol 29: 2376-2384, 1999.

14. Riccioni R, Rossini A, Calabro L, Diverio D, Pasquini L, Lococo F, Peschle C and Testa U: Immunophenotypic features of acute myeloid leukemias overexpressing the interleukin 3 receptor alpha chain. Leuk Lymphoma 45: 1511-1517, 2004.

15. Corbel C, Lemarchandel V, Thomas-Vaslin V, Pelus AS, Agboton C and Romeo PH: Neuropilin 1 and CD25 co-regulation during early murine thymic differentiation. Dev Comp Immunol 31: 1082-1094, 2007.

16. Pries R and Wollenberg B: Cytokines in head and neck cancer. Cytokine Growth Factor Rev 17: 141-146, 2006.

17. Pries R, Thiel A, Brocks C and Wollenberg B: Secretion of tumorpromoting and immune suppressive cytokines by cell lines of head and neck squamous cell carcinoma. In Vivo 20: 45-48, 2006.

18. Waugh DJ and Wilson C: The interleukin-8 pathway in cancer. Clin Cancer Res 14: 6735-6741, 2008.

19. Chaput N, Taieb J and Zitvogel L: [Innate defence against tumor cells: the killer cell IKDC]. Bull Cancer 93: 449-451, 2006.

20. Papewalis C, Jacobs B, Wuttke M, et al: IFN-alpha skews monocytes into CD56+-expressing dendritic cells with potent functional activities in vitro and in vivo. J Immunol 180: 1462-1470, 2008.

21. Buhring HJ, Simmons PJ, Pudney M, Muller R, Jarrossay D, van Agthoven A, Willheim M, Brugger W, Valent P and Kanz L: The monoclonal antibody 97A6 defines a novel surface antigen expressed on human basophils and their multipotent and unipotent progenitors. Blood 94: 2343-2356, 1999.

22. Goding JW: Ecto-enzymes: physiology meets pathology. J Leukoc Biol 67: 285-311, 2000.

23. Yano Y, Hayashi Y, Sano K, Nagano H, Nakaji M, Seo Y, Ninomiya T, Yoon S, Yokozaki $\mathrm{H}$ and Kasuga M: Expression and localization of ecto-nucleotide pyrophosphatase/phosphodiesterase I-1 (E-NPP1/PC-1) and -3 (E-NPP3/CD203c/PD-Ibeta/ B10/gp130(RB13-6)) in inflammatory and neoplastic bile duct diseases. Cancer Lett 207: 139-147, 2004.

24. Yano Y, Hayashi Y, Sano K, Shinmaru H, Kuroda Y, Yokozaki H, Yoon S and Kasuga M: Expression and localization of ecto-nucleotide pyrophosphatase/phosphodiesterase I-3 (E-NPP3/CD203c/PD-I beta/B10/gp130RB13-6) in human colon carcinoma. Int J Mol Med 12: 763-766, 2003. 